\title{
Fibroblast growth factor 2 and the transcription factor Egr-I localise to endothelial cell microvascular channels in human coronary artery occlusion
}

A 69-year old male with angina, hypertension, hyperlipidemia and Type II diabetes mellitus underwent percutaneous coronary intervention (PCI) to a 1-2 month old total occlusion of the right coronary artery (RCA) (Fig. 1A-C). In an uncomplicated and successful procedure, a distal protection device (Filter Wire EX, Boston Scientific, Natick, MA, USA) was used to minimise peri-procedural distal embolisation into viable myocardium (Fig. 1B). Immunohistopathology of the recovered material forms this report.

Following the procedure, material from the device was placed in $10 \%$ neutral buffered formalin, and paraffin embedded. Haematoxylin and Eosin and Masson's Trichrome stains were performed on $5 \mu \mathrm{m}$ serial sections. In addition, the following were examined using specific antibodies: the transcription factors early growth response factor-1 (Egr-1) and c-jun (Santa Cruz Biotechnology, Santa Cruz, CA, USA); the growth factors Fibroblast Growth Factor 2 (FGF-2) and Vascular Endothelial Growth Factor (VEGF) (Santa Cruz Biotechnology), Proliferating Cell Nuclear Antigen (PCNA) (Dako, Carpinteria, CA, USA), Tissue Factor (TF) (gift from Prof. Wolfram Ruf, Scripps Research Institute La Jolla, CA, USA) and the endothelial cell marker CD31 (Santa Cruz Biotechnology). Secondary antibodies were 3-amino-9-ethylcarbazole (AEC) for CD 31 and PCNA, and 3,3'-diaminobenzadine (DAB) for Egr-1, c-jun, FGF-2, TF and VEGF, respectively.

The tissue fragments measured up to $4.2 \mathrm{~mm}$ in length and up to $1.5 \mathrm{~mm}$ in diameter. They were composed of fibrin and a small amount of newly deposited collagenous connective tissue, together with neovascular channel formation throughout the tissue (Fig. 1D, E), suggestive of organising thrombus. The vascular channels were comprised of a single layer of endothelial cells, confirmed by positive staining for CD31 (Fig. 1F), which were PCNA positive (Fig. 1G) suggesting active proliferation. From the extent of neovascular channel formation, and the degree of proliferation, it is estimated this organising thrombus was several weeks old. This is consistent with the clinical evidence, which placed the occlusion at 1-2 months old on angiographic grounds. The cells also showed some positive staining for Egr-1 (predominantly nuclear) and more markedly positive staining for FGF-2 (mainly nuclear and cytoplasmic) (Fig. 1H), though not for c-jun, VEGF or TF (data not shown). There was minimal staining for Egr-1 or FGF-2 elsewhere in these lesions.

\footnotetext{
Correspondence to:

Harry C. Lowe

Centre for Vascular Research

School of Medical Sciences

University of New South Wales

Sydney NSW 2052, Australia

Tel: 6 I 29767 6296, Fax : 61297676994

E-mail: h.lowe@unsw.edu.au

Received July 14, 2004

Accepted after resubmission October 25, 2004
}

Thromb Haemost 2005; 93: 172-4
Chronic total coronary occlusions remain a problematic area in cardiology, with limited available treatments (1). Totally occluded coronary arteries are known to contain multiple microvascular channels after 3 months (2), which may progress to recanalisation, providing alternative pathways for antegrade blood flow and symptom relief. However, the mechanisms by which these channels form are unknown, and little is known of the histology prior to 3 months.

These data demonstrate, for the first time, localisation of FGF-2 and Egr-1 protein to endothelial cells forming microvascular channels throughout organising thrombus, 1-2 months following human coronary artery occlusion.

FGF-2 (or basic FGF) is a polypeptide growth factor which modulates a number of cell functions including angiogenesis, vasculogenesis, cell proliferation and migration in a variety of cell types, but with particular emphasis on endothelial cells (3). It is a member of the larger fibroblast growth factor family, which contains over 20 structurally related polypeptides $(3,4)$. FGF-2 lacks a classical signal peptide, and although cellular release of the protein can occur via a number of other mechanisms, the actions of FGF-2 can occur via both intracellular and extracellular means $(3,4)$. These functions include activation of key transcription factor pathways. Egr-1 is a pleiotropic transcription factor, and a key upstream activator of a number of pathways mediating vascular cell proliferation, migration adhesion and coagulation (5).

The finding here of FGF-2 and Egr-1 up-regulation in endothelial cell microvascular channels within organising thrombus is intriguing. Clearly, co-localisation does not prove an association of function, and the limited tissue fragments available prevented more definitive co-localising studies. However, endothelial cell FGF-2 dependent Egr-1 transcription is known to occur in response to vascular injury (6). Similar FGF-2/Egr-1 dependent mechanisms have recently been invoked in the context of angiogenesis in neovascularisation and tumour growth (7). Given these observations, the present data suggest FGF-2 and Egr-1 may be involved in an endothelial gene regulatory circuit involved in the formation of these microvascular channels, and that similar processes may be involved in thrombus neovascularisation in the human coronary artery. The observation that these endothelial channels show PCNA positive staining also suggests that any FGF-2 and Egr-1 dependent processes are playing a role during the active, early proliferating phase of neovascularisation.

Whether FGF-2 regulates Egr-1 or vice-versa, or both, is not clear. Our data, nonetheless, indicate that coronary occlusions, rather than being an end-stage process, are active and undergo dynamic change, and suggest FGF-2 and Egr-1 may play a role in this process.

In contrast, VEGF and TF staining were negative. VEGF is a potent inducer of angiogenesis (8), and is known to play a role in 
Figure I: Immunohistopathology of material collected during percutaneous treatment of occluded right coronary artery. Coronary angiography, left anterior oblique (LAO) projection: A. Right coronary artery occlusion indicated at arrow; B. Balloon dilatation and stent deployment with distal protection device in-situ (arrow); C. Final angiographic result. Histopathology of material collected in distal protection device: $\mathrm{D}$. Haematoxylin and Eosin stain (x 40) demonstrating neovascular channels (arrows) in the organising thrombus; an intravascular neutrophil is identifiable (labelled N); E. Masson's Trichrome stain (x 40) demonstrating neovascular channels (arrows) in longitudinal and cross section, in a matrix comprising a mixture of collagen fibres (blue) and fibrin (red). Immunohistochemical analysis of material collected: Neovasular channels within the organising thrombus stained using antibodies directed to: (F) CD3 I $(4 \mu \mathrm{g} /$ $\mathrm{ml})$, (G) PCNA(I0 $\mu \mathrm{g} / \mathrm{ml})$, (H) Egr-I $(20 \mu \mathrm{g} / \mathrm{ml})$, (I) FGF-2 $(2 \mu \mathrm{g} / \mathrm{ml})$, magnification $x 40$. Hematoxylin counterstain for CD3I studies. Note predominantly nuclear staining for (G) PCNA and $(\mathrm{H})$ egr-I; nuclear and cytoplasmic staining for (F) CD3I and (I) FGF-2.
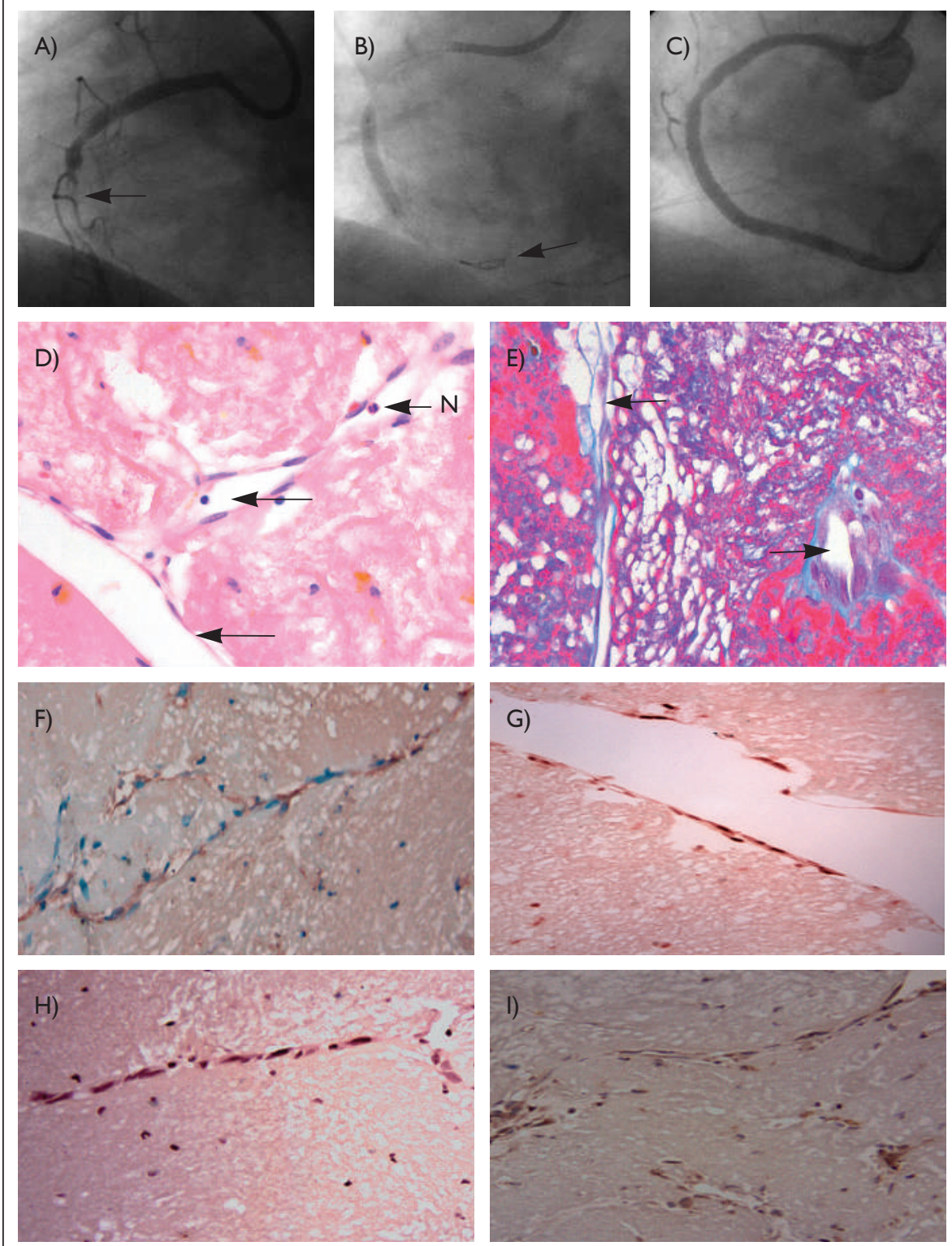

recanalisation of venous thrombosis (9). Its role in recanalisation of arterial thrombus, and in arterial collateral formation however, is less clear $(8,10)$. Likewise, TF was not seen to be upregulated. TF expression is increased by Egr-1 in a number of contexts, and plays a role in thrombus formation (11). The negative findings here clearly do not preclude roles for VEGF or $\mathrm{TF}$, but rather highlight the need for further examination of tissue obtained in this way, from similar patients.

These observations raise a number of additional issues. Firstly, given prior human experience of intracoronary FGF delivery (12), the potential for FGF to provide pro-angiogenic, therapeutic benefit for total occlusions may become a consideration. Second, the demonstration of large tissue fragments trapped in the distal protection device, suggests that significant distal embolisation was prevented and that these devices may have a place in the treatment of chronic coronary total occlusions. How often material collected in this way is useful for pa- thologic studies however, is at present unknown. Finally, whilst the present data are limited to a single patient, such observations may prompt further investigation into local vascular reparative mechanisms following total coronary occlusion, potentially providing novel therapeutic options.

\section{Acknowledgements}

This work is supported by the National Heart Foundation of Australia (RB), the National Health and Medical Research Council of Australia (LMK and HCL) and a Pfizer Cardiovascular Lipid Grant (HCL, DB).

Ravinay Bhindi',2, David Brieger2, Hideto Ishii', Nick Di Girolamo3, Rakesh K. Kumar', Levon M. Khachigian', Harry C. Lowe',2

'Centre for Vascular Research, ${ }^{3}$ Department of Pathology, School of Medical Sciences, University of New South Wales, Sydney, Australia, ${ }^{2}$ Cardiology Department, Concord Repatriation General Hospital, Sydney, Australia 


\section{References}

1. Bailey SR. Opportunity lost: the continuing challenge of chronic total occlusions. Catheter Cardiovasc Interv 2003; 59: 312-3.

2. Srivatsa SS, Edwards WD, Boos CM, et al. Histologic correlates of angiographic chronic total coronary artery occlusions: influence of occlusion duration on neovascular channel patterns and intimal plaque composition. J Am Coll Cardiol 1997; 29: 955-63.

3. Szebenyi G, Fallon JF. Fibroblast growth factors as multifunctional signaling factors. Int Rev Cytol 1999; 185: 45-106.

4. Detillieux KA, Sheikh F, Kardami E, et al. Biological activities of fibroblast growth factor-2 in the adult myocardium. Cardiovasc Res 2003; 57: 8-19.

5. Khachigian LM, Collins T. Inducible expression of Egr-1-dependent genes. A paradigm of transcriptional activation in vascular endothelium. Circ Res 1997; 81: 457-61.

6. Santiago FS, Lowe HC, Day FL, et al. Early growth response factor-1 induction by injury is triggered by release and paracrine activation by fibroblast growth factor-2. Am J Pathol 1999; 154: 937-44.

7. Fahmy RG, Dass CR, Sun LQ, et al. Transcription factor Egr-1 supports FGF-dependent angiogenesis during neovascularization and tumor growth. Nat Med 2003; 9: 1026-32

8. Neufeld G, Cohen T, Gengrinovitch S, et al. Vascular endothelial growth factor (VEGF) and its receptors. FASEB 1999; 13: 9-22.

9. Waltham M, Burnand KG, Collins M, et al. Vascular endothelial growth factor enhances venous thrombus recanalisation and organisation. Thromb Haemost 2003; 89: 169-76.
10. Deindl E, Buschmann I, Hoefer IE, et al. Role of ischemia and of hypoxia-inducible genes in arteriogenesis after femoral artery occlusion in the rabbit. Circ Res 2001; 89: 779-86.

11. Yan SF, Mackman N, Kisiel W, et al. Hypoxia / Hypoxemia Induced activation of the procoagulant pathways and the pathogenesis of ischemia-associated thrombosis. Arterioscler Thromb Vasc Biol 1999; 19 2029-35.

12. Aviles RJ, Annex BH, Lederman RJ. Testing clinical therapeutic angiogenesis using basic fibroblast growth factor (FGF-2). Br J Pharmacol 2003; 140: $637-46$. 